\title{
Scintillation and pupil illumination in AO coronagraphy
}

\author{
Anand Sivaramakrishnan ${ }^{1,6}$, B. R. Oppenheimer ${ }^{1}$, M. D. Perrin ${ }^{2,6}$, \\ L. C. Roberts, Jr ${ }^{4}$., R. B. Makidon ${ }^{3,6}$, R. Soummer ${ }^{1,6}$ A. P. Digby ${ }^{1}$, \\ L. W. Bradford ${ }^{4}$, M. A. Skinner ${ }^{4}$, N. H. Turner ${ }^{5}$, \\ and T. A. ten Brummelaar ${ }^{5}$ \\ ${ }^{1}$ Department of Astronomy, American Museum of Natural History, 79th Street at Central \\ Park West, New York NY 10024, USA \\ ${ }^{2}$ Astronomy Department, University of California, Berkelely CA 94000, USA \\ ${ }^{3}$ Space Telescope Science Institute, 3700 San Martin Drive, MD 21218, USA \\ ${ }^{4}$ The Boeing Company, 535 Lipoa Pkwy, Suite 200, Kihei, HI 96753, USA \\ ${ }^{5}$ Center for High Angular Resolution Astronomy, Georgia State University Mt. Wilson CA \\ 91023, USA \\ ${ }^{6} \mathrm{NSF}$ Center for Adaptive Optics
}

\begin{abstract}
The Lyot Project near-infrared $J H K$ coronagraph achieved first light on the Advanced Electro-Optical System (AEOS) in March 2004. Optical pupil plane imaging at video rates from this coronagraph provides data on atmospheric scintillation and quasi-static pupil intensity variations. We examine the effect of these variations on coronagraphic performance. Early simulations suggested Strehl ratio reductions of the order of $2-3 \%$ due to residual uncorrected phase aberrations in $H$-band. We find that static or quasi-static pupil illumination non-uniformity in $I$-band reduces Strehl by $\sim 2 \%$. A lower bound on the effects of dynamic illumination variation over the pupil is also $\sim 2 \%$ in $I$-band. Some of the static intensity variations in the pupil are due to pinned deformable mirror (DM) actuators. We simulate the effects a pinned actuator has on the coronagraph. The resultant speckles in simulated coronagraphic images show similarities to some Lyot Project PSFs. This highlights the importance of knowledge of the pupil in next-generation extreme AO coronagraphs in order to realize the predicted photometric dynamic range of their images.
\end{abstract}

Keywords. instrumentation: adaptive optics, planetary systems: protoplanetary disks.

\section{Introduction}

The 3.6 m AEOS adaptive optics (AO) system's 941-element deformable mirror (DM) provides higher order wave front correction than any other astronomical AO system today. It produces images with Strehl ratios $(\mathrm{SRs})$ of $\sim 0.15$ in the $I$-band for objects brighter than 6 th magnitude, with SR's as high as $\sim 0.25$ during the best seeing conditions (Roberts \& Neyman 2002). The Lyot Project near-infrared coronagraph (Oppenheimer et al. 2004), at its coudé focus, delivers $H$-band SRs in the $\sim 0.65-0.83$ range (Perrin et al. 2004, Makidon et al. 2005), and occasionally higher. This performance agrees in the main with predictions based on results from simple AO simulations anchored in Palomar and AEOS AO data (Sivaramakrishnan et al. 2001, Makidon et al. 2005), but dynamic range with the coronagraph still falls short of predictions based on numerical modelling. Incorporating knowledge of pinned actuators on the deformable mirror (DM) into simulations brings modelling and coronagraphic data into closer agreement. 


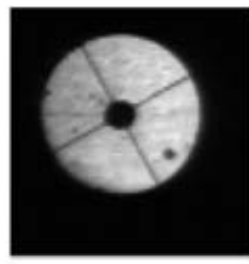

$a$
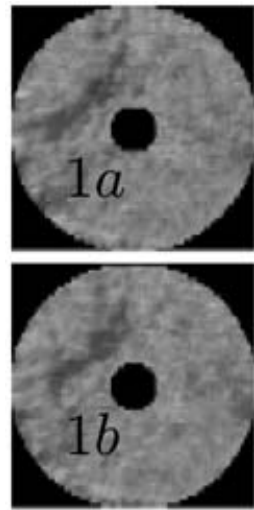

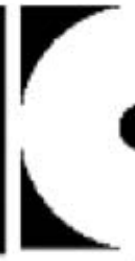

$b$
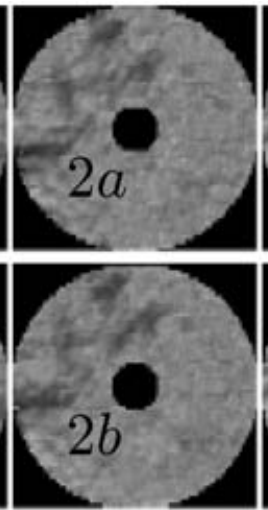

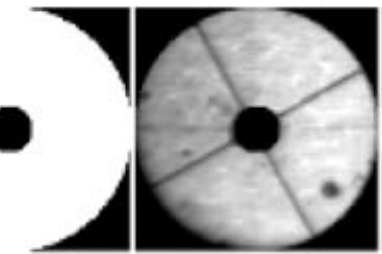

$c$
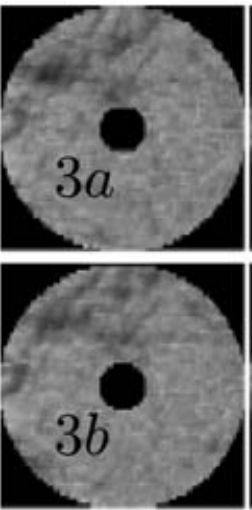

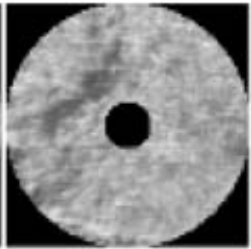

$d$
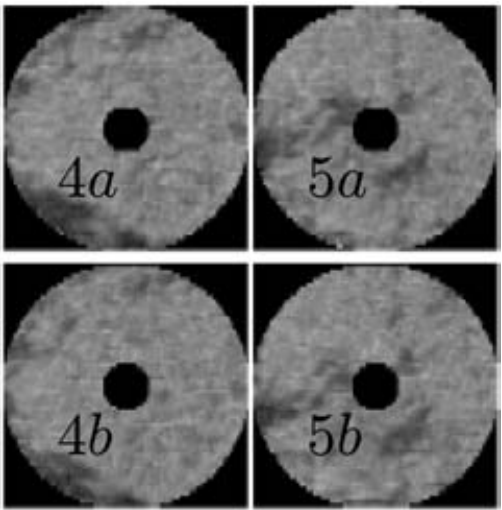

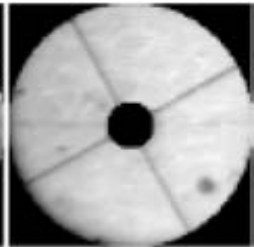

e

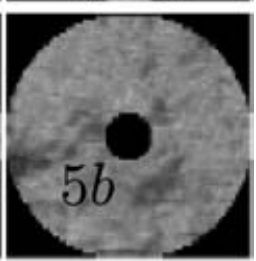

Figure 1. Raw and processed video rate CCD images of the pupil (see text).

\section{Observations, calibration and theory}

The Lyot Project's pupil-viewing mode uses a video rate CCD to record the intensity distribution of light in the pupil (Bradford et al. 2005). These images were taken in a non-coronagraphic mode, with the focal plane stop (which at $H$ is a $4 \lambda / D$ diameter hole in a mirror) moved out of the beam. While the SR is reduced slightly because of pinned DM actuators on the DM, the localized nature of the resulting aberration produces image plane speckles which are not suppressed by a coronagraph. This decreases the dynamic range of the coronagraph. Normally an interlaced video-rate CCD camera observes light that is rejected by a Lyot mask (at a reimaged pupil). Using a flat mirror instead of this mask, we collected full pupil images, in $176 \times 180$ pixel frames with a $9 \mu \mathrm{m}$ pixel pitch. The illuminated pupil is approximately 116 pixels, so each pixel is $3.12 \mathrm{~cm}$ across on the pupil, or 3.64 pixels per AO system subaperture $(11.3 \mathrm{~cm})$. The CCD is read using 12-bit digitization. The AO system was not actively correcting atmospheric aberrations during this experiment. We present data from one observation of a $V=2.9$ star, HR 7235 (spectral class A0V, with a parallax of 39.18 mas), on 8 June 2004. The rising star was at a zenith distance of $13^{\circ}$ and an azimuth of $122^{\circ}$ at the start of observations. We obtained 2048 frames at $30 \mathrm{~Hz}$ in a little over a minute elapsed time.

\subsection{Calibration}

When the CCD is evenly illuminated it shows no detectable features or pixel to pixel variations that are larger than $1: 10^{3}$. We therefore used the data frames without flatfielding the detector response. Bias subtraction was done with un-illuminated regions of sets of 512 frames; the median of 16 dark columns on either end of the detector was subtracted from each full frame. After bias subtraction, mean and median frames of the stack were computed. To compensate for pupil wander (a single CCD frame is shown in Fig. 1a, on a different scale than all the other pupil images in this paper), the images 


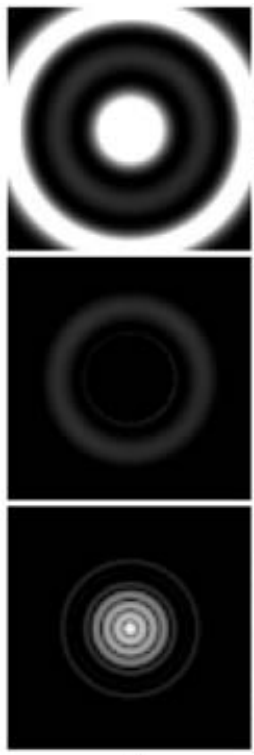

$a$
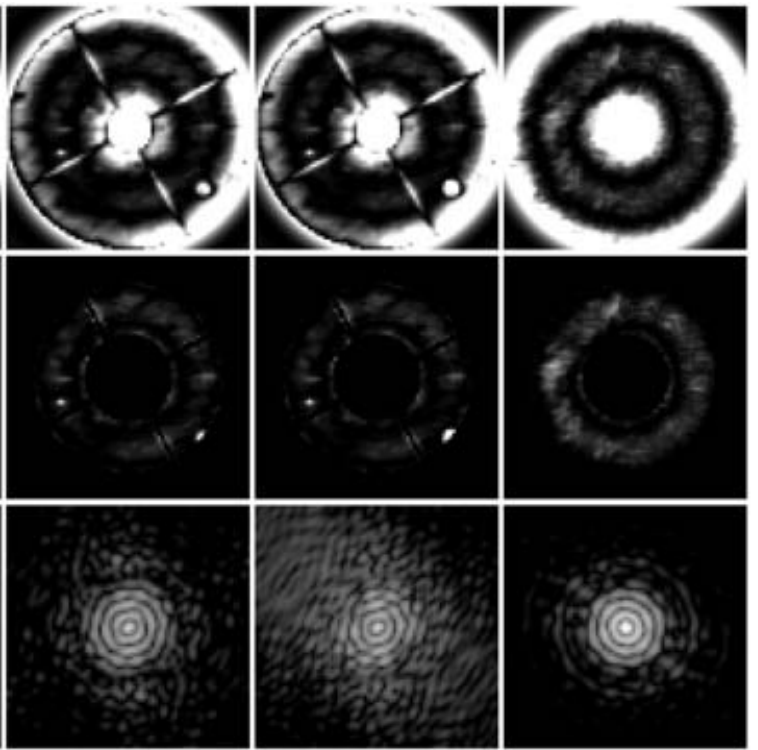

$b$

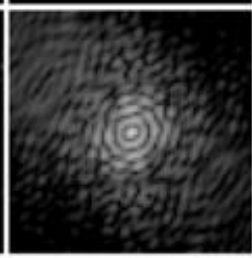

$c$

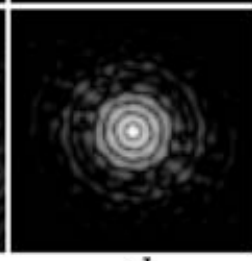

$d$

Figure 2. Lyot pupil and coronagraphic image intensities (see text).

were registered to the nearest pixel by cross-correlation with a numerical pupil mask representing the centered pupil, with outside diameter 116 pixels, inside diameter 22 pixels (Fig. 1b).

Secondary support spiders are narrower than our projected pixel scale, and are not precisely in a pupil plane. They partially vignette our pupil image. This can be calibrated out by "illumination-flat-fielding". The normalized mean of stacked, registered, images is the "illumination flat" (Fig. 1c). Each bias-subtracted data frame in the stack was divided by this flat (e.g. Fig. 1d) - these video rate frames measure the atmospheric scintillation. The square root of the flat field (Fig. 1e) is the quasi-static pupil illumination function $A_{o}(\mathbf{x})$ of the coronagraph. The scintillation frames were then de-interlaced by creating two sub-frames (labelled $a$ and $b$ ) from each parent frame. Five pairs of sub-frames from successive parent frames are shown in Fig. 1 (bottom). Inter-frame timing between the $a$ and $b$ sub-frames is tightly controlled by CCD camera hardware. Operating system characteristics prevented reliable timing between succesive full frames of the CCD.

\subsection{Non-uniform Pupil Illumination Effects in Coronagraphy}

We describe pupil illumination non-uniformity with the real function

$$
A(\mathbf{x}, t)=A_{o}(\mathbf{x}) f(t)(1+Z(\mathbf{x} ; t))
$$

where $t$ represents time, and $\mathbf{x}$ the pupil plane position vector. The real function $Z(\mathbf{x} ; t)$ is constrained to possess a zero mean over the clear aperture at any instant. $A(\mathbf{x}, t)$ is the modulus of the complex-valued pupil field strength. We do not concern ourselves with the photometric ramifications of scintillation (e.g. Dravins et al. 1998), since direct detection and, if possible, spectroscopy are our focus. We subsume the temporal variation of the net power entering the telescope into the function $f(t)^{2}\left(1+Z(\mathbf{x} ; t)^{2}\right)$. The two-dimensional spatial frequency content of instantaneous 'realizations' of $Z(\mathbf{x} ; t)$ cause scintillation to be a concern for ground-based near-IR coronagraphy. In the weak scintillation regime in the $H$-band, $Z^{2} \ll 1$. We use observed $I$-band pupil intensities as input to our models. 

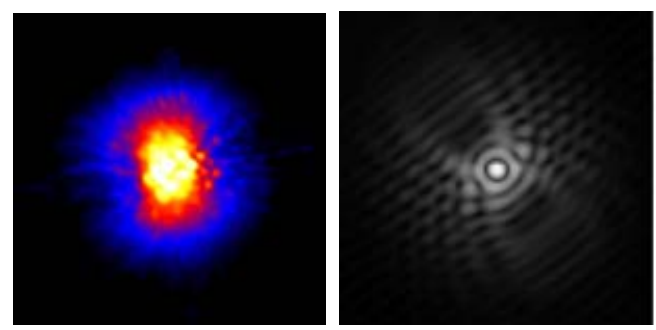

Figure 3. Vega ( $8 \mathrm{~s}, \mathrm{H}$-band) taken with the Lyot Project coronagraph (left). The pixel scale is 13.5 mas (the image is $3^{\prime \prime} .2$ across). Elongation of the PSF is due to pinned actuators on the AO system's DM. The phase aberration induces $\sim 10 \%$ reduction in Strehl ratio. A simulated H-band coronagraphic image with five pinned actuators on the DM (right).

\section{Conclusion}

In Fig. 2 we show the Lyot pupil intensity before (top row) and after (middle row) the Lyot stop, and the coronagraphic PSF (log scale, bottom row) simulated in the $H$-band. We simulate uniform pupil illumination (column $a$ ), the observed quasi-static intensity without $(b)$ and with $(c)$ phase errors from a pinned actuator 1.25 microns below the DM surface, and only atmospheric scintillation sub-frame $(d)$. The pinned actuator's phase errors contribute the most speckle noise to the image by far. Fig. 3 shows real data (left) and a simulated image with five pinned actuators on the DM (right). The morphology of the actual coronagraphic PSF's core and inner regions is reproduced qualitatively by this model.

\section{Acknowledgements}

This research was supported by the U.S. Air Force Office of Scientific Research through AFRL/DE (Contract Number F29601-00-D-0204), the U.S. National Science Foundation (NSF) through grants No. AST-0088316 and AST-0215793, the NSF Science and Technology Center for Adaptive Optics, managed by the University of California at Santa Cruz under cooperative agreement AST-9876783, the Cordelia Corporation, Hillary Lipsitz, an anonymous supporter, and Space Telescope Science Institute's Research Programs Office and Director's Discretionary Research Fund. Soummer and Digby are supported by NASA Michelson Postdoctoral fellowships, and Perrin by a NASA Michelson Graduate Fellowship, under contract to the Jet Propulsion Laboratory (JPL) funded by NASA. The JPL is managed for NASA by the California Institute of Technology.

\section{References}

Bradford, L. W., Roberts, L. C., Skinner, M. A., Digby, A. P., Oppenheimer, B. R., Perrin, M. D., \& Turner, N. H. 2005, AMOS Tech. Conf.

Dravins, D., Lindegren, L., Mezey, E., \& Young, A. T. 1998, PASP, Vol. 110, 610

Lyot, B. 1939, MNRAS, Vol. 99, 580

Makidon, R. B., Sivaramakrishnan, A., Perrin, M. D., Roberts, L. C., Jr., Oppenheimer, B. R., Soummer, R., \& Graham, J. R. 2005, PASP Vol. 117, 831

Oppenheimer, B. R. et al. 2004, Proc. SPIE Vol. 5490

Perrin, M. D., Graham, J. R., Trumpis, M., Kuhn, J., Whitman, K., Coulter, R., Lloyd, J. P., \& Roberts, L. C., Jr. 2004, 2003 AMOS Tech. Conf.

Roberts, L. C., Jr. \& Neyman, C. R. 2002, PASP Vol. 114, 1280

Sivaramakrishnan, A., Koresko, C. D., Makidon, R. B., Berkefeld, T., \& Kuchner, M. J. 2001, ApJ Vol. 552, 397 